\title{
A Self-Grounded Dual-Polarized Wideband Bowtie With Improved MIMO Performance in Random-LOS
}

\author{
Sadegh Mansouri Moghaddam ${ }^{1}$, Per-Simon Kildal ${ }^{1}$, Andrés Alayón Glazunov ${ }^{1}$, Jian Yang ${ }^{1}$, Mattias Gustafsson ${ }^{2}$ \\ ${ }^{1}$ Department of Signal and Systems, Chalmers University of Technology \\ ${ }^{2}$ Huawei Technologies Sweden AB \\ Gothenburg, Sweden \\ sadegh.mansouri@chalmers.se,per-simon.kildal@chalmers.se, andres.glazunov@chalmers.se,jian.yang@chalmers.se, \\ mattias.gustafsson@huawei.com
}

\begin{abstract}
We present a new improved design of the selfgrounded dual-polarized wideband bowtie antenna for MIMO application. The antenna is evaluated for the case when the original four ports are combined to two orthogonally polarized ports. The new antenna covers the previous bandwidth but has lower gain and wider beamwidth over the frequency band of interest. The $2 \times 2$ MIMO performance of the antenna in RandomLine of Sight (Random-LOS) is evaluated for two bitstreams using the threshold receiver model and Zero-Forcing receiver. The simulated results show an improvement in terms of MIMO multiplexing coverage over a hemisphere for two bitstreams, compared to the previous solution.
\end{abstract}

Keywords-MIMO; Random-LOS; self-grounded Bowtie.

\section{INTRODUCTION}

The increasing demand for communication systems with higher capacity and data rata has made an interest in MIMO technology [1]. Making use of multiple antennas at both the transmitter and receiver sides can increase the capacity of the channel and improve the reliability of the system without increasing the input power or frequency bandwidth. Having the constraint of compactness and high isolation between antenna ports calls for the use of polarization-MIMO, where two orthogonal polarizations are used as MIMO subchannels [2]. Almost all the previous studies on polarization-MIMO analyzed the antenna in a multipath environment. However, there might be a LOS component between the transmitter and the receiver and this contribution will increase at higher frequencies. Therefore, a characterization method to evaluate the performance of the antenna in a pure LOS propagation condition becomes essential. However, this LOS component is random in terms of Angle-of-Arival (AoA) and polarization due to the randomness of location and orientation of the user side. This we call Random Line Of Sight (Random-LOS). The Random-LOS characterization of a bowtie micro-basestation antenna for MIMO systems was introduced in [3]. We discovered in [4] a performance degradation of this self-grounded bowtie antenna when it was excited for two orthogonally polarized ports in Random-LOS. In the current paper, we improve the performance of the previously designed antenna in RandomLOS by simulations using CST Microwave Studio. The Probabilities of Detection (PoD) are obtained by postprocessing using the MATLAB-based simulation tool named Virmlab [5], and the threshold receiver model [6]. Some PoD performance

The present work has been supported by the Swedish Governmental Agency for Innovation Systems (VINNOVA) within the VINN Excellence Center Chase at Chalmers, and by the same VINNOVA with a project on MIMO hardware within the Innovative ICT 2013 program. characteristics of polarization-MIMO in Random-LOS is given in [7].

\section{GeOMETRIES, $\mathrm{S}_{11}$ AND DIRECTIVITIES}

The geometry of two different designs of the self-grounded dual-polarized bowtie antenna are shown in Fig.1. Both antennas consist of 4 petals attached to the ground. Two opposite petals are, in this paper, excited differentially which results in two orthogonally polarized ports. In the previous design (top figure), the petals are bended over half a virtual cylinder [8]. In the improved design (bottom figure) the bending procedure over virtual cylinder is changed and the bending is performed over $5 / 8$ of a cylinder. This new bending approach will gives us more freedom to control the length of the petals which comes straight out of the feeding point and makes the structure more flexible to optimize the radiation pattern in a desirable manner. The heights (H) are $29.5 \mathrm{~mm}$ and $33.6 \mathrm{~mm}$ and the largest dimensions (W) are $111 \mathrm{~mm}$ and $104 \mathrm{~mm}$ for the previous and new designs, respectively. The simulated reflection coefficients and directivities of both designs are shown in Fig. 2a. As can be seen, the reflection coefficient is below $-10 \mathrm{~dB}$ within 1.6 to $3 \mathrm{GHz}$ for both cases. However, the previous design has lower reflection coefficient at the lower frequencies, but the new design has larger $-10 \mathrm{~dB}$ bandwidth. The directivity is $5-6.5 \mathrm{dBi}$ and 2.9 $4.3 \mathrm{dBi}$ for the previous and new structures, respectively (Fig.2b). Hence, while the previous antenna is more directive, the new design has a wider beamwidth which, as we going to show next, results in better MIMO performance in the desired $+/-90^{\circ}$ angular coverage.
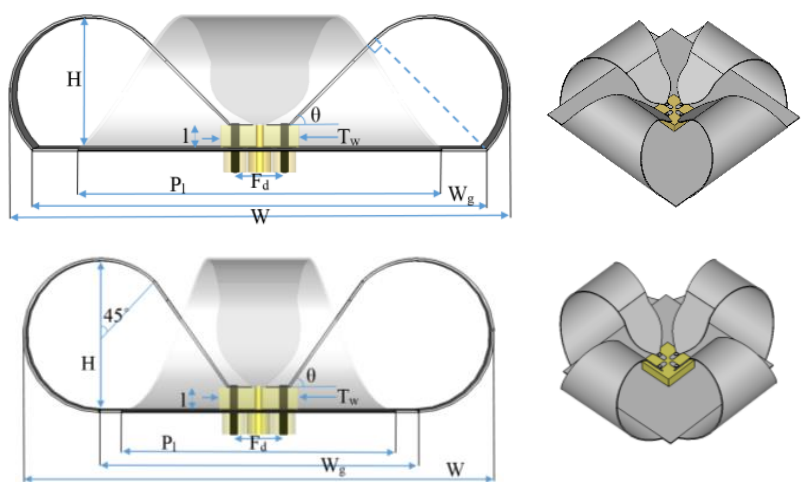

Fig. 1: Side view (left) and 3D view (right) of previous (upper) and new (lower) self-grounded dual-polarized bowtie antenna. 


\section{MIMO PERFORMANCE}

After plotting PoD curves, the MIMO multiplexing efficiency can be determined by comparing the PoD with the appropriate reference PoD curve at the $95 \%$ PoD level. We use here a reference corresponding to a hemispherical isotropic polarization-matched coverage for 2-bitstreams. So the reference is actually an ideal hemispherically isotropic two-port dual-polarized antenna with $100 \%$ efficiency and completely orthogonal polarizations for ports in whole hemispherical coverage.

The MIMO efficiency of both designs are shown in Fig. 3 versus frequency and the desired coverage in degrees. The coverage angle is here given as a polar angle from the center of the beam. Coverages corresponding to $30^{\circ}$ and $60^{\circ}$ are illustrated by circles in the coverage plots in Fig. 4. The desired shape is to have the same coverage within the whole frequency band, and as high level as possible within the desired coverage angle. We see in Fig. 3 that, there is a drop in the 2-bitstream MIMO efficiency at $2.2 \mathrm{GHz}$ in the previous design, and that is almost removed in the new design. In the improved case, the MIMO efficiency is around $-10 \mathrm{dBi}$ with respect to the hemispherically isotropic reference, i.e. with $90^{\circ}$ coverage cone. This means that $95 \%$ percent of the time, we have hemispherical coverage with at most $10 \mathrm{~dB}$ increased signal power compared to the hemi-isotropic reference. This is about $15 \mathrm{~dB}$ improvement with respect to the previous antenna for this coverage.

Calculating relative received power of each bitstream for each AoA by using ZF-algorithm at receiver side, the 2bitstream MIMO throughput coverage pattern can be plotted as shown in Fig. 4. Note that, the two incoming waves have fixed orthogonal polarizations (horizontal and vertical). We see that for the previous design, the coverage degrades drastically for angular coverage larger than $60^{\circ}$ at the middle frequencies. In the other hand, the new antenna has almost flat coverage over the whole hemisphere.
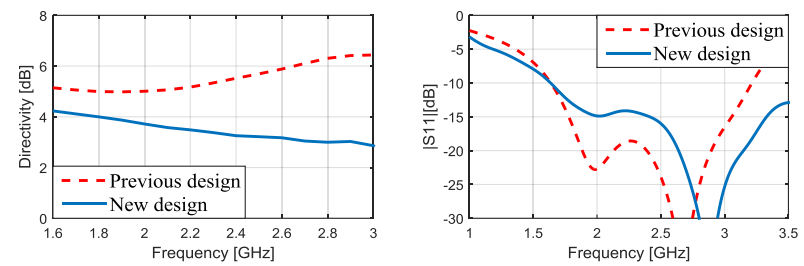

Fig. 2 : Simulated directivities and reflection coefficients of both previous and new self-grounded dual-polarized bowtie antenna.
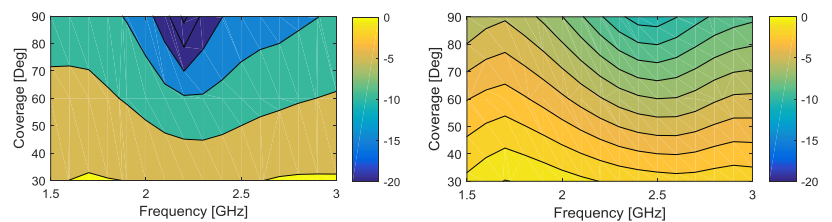

Fig. 3 : 2-bitstream MIMO efficiency in $\mathrm{dBi}$ in random-LOS for different coverages at different frequencies for previous (left) and new antenna (right). Coverage is considered on a cone around the axis normal to the ground planes.

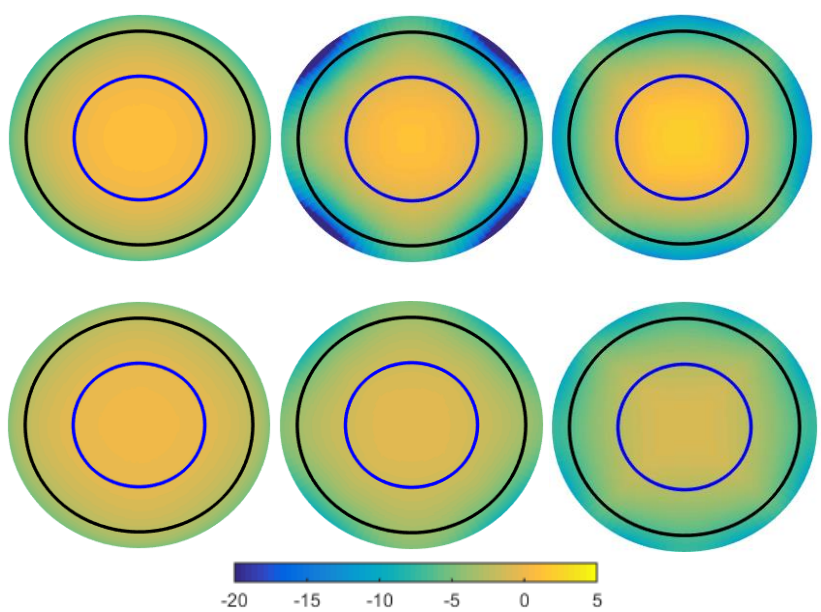

Fig. 4 : 2-bitstream MIMO throughput coverage of previous (upper) and new (lower) antenna at the frequencies of $1.7 \mathrm{GHz}$ (left), 2.2 GHz (middle) and $2.7 \mathrm{GHz}$ (right). Incoming waves are two orthogonal linearly polarized. Blue and black circles correspond to $30^{\circ}$ and $60^{\circ}$ coverage cones, respectively.

\section{CONCLUSION}

A new version of the self-grounded dual-polarized bowtie antenna was designed to cancel out the performance degradation in the middle of the band of a previous bowtie in terms of MIMO multiplexing efficiency coverage in Random-LOS.

\section{REFERENCES}

[1] D. Gesbert, M. Shafi, D.-S. Shiu, P. Smith and A. Naguib, "From theory to practice: An overview of MIMO space-time coded wireless systems," IEEE J. Sel. Areas Commun., pp.281-302,2003.

[2] N. K. Das, T. Inoue, T. Taniguchi, and Y. Karasawa, "An experiment on MIMO system having three orthogonal polarization diversity branches in multipath-rich environment," in Proc. IEEE $60^{\text {th }}$ Vehicular Technol. Conf., vol. 2, pp. 1528-1532 ,Sep. 2004.

[3] P.-S. Kildal, X. Chen, M. Gustafsson, Z. Shen, "MIMO Characterization on System Level of $5 \mathrm{G}$ Micro Base Stations Subject to Randomness in LOS", IEEE Access, Vol. 22, pp. 1062-1075, Sept. 18, 2014

[4] S. Mansouri, A.A. Glazunov, J. Yang, M. Gustafsson and P.-S. Kildal, "Comparison of 2-bitstream polarization-MIMO performance of 2 and 4 port Bowtie antenna for LTE in Random-LOS" (presented ISAP2015).

[5] U. Carlberg, J. Carlsson, A. Hussain, and P.-S. Kildal, "Ray based multipath simulation tool for studying convergence and estimating ergodic capacity and diversity gain for antennas with given far-_eld functions," in Proc. 20th Int. Conf. Appl. Electromagn. Commun. (ICECom), Dubrovnik, Kroatia, pp. 1_4, Sep. 2010 (Virmlab is available for downloading at www.kildal.se )

[6] A. Razavi, A. Alayón Glazunov, P.-S. Kildal, J. Yang, "Characterizing Polarization-MIMO Antennas in Random-LOS Propagation Channels", submitted to IEEE AWPL, Dec 2015.

[7] P. S. Kildal, A. Hussain, X. Chen, C. Orlenius, A. Skarbratt, J. Asberg, T. Svensson, and T. Eriksson, "Threshold Receiver Model for Throughput of Wireless Devices With MIMO and Frequency Diversity Measured in Reverberation Chamber," IEEE Antennas and Wireless Propagation Letters, vol. 10, pp. 1201-1204, 2011.

[8] H. Raza, A. Hussain, J. Yang, and P.-S. Kildal, "Wideband compact 4 port dual polarized self-grounded bowtie antenna," IEEE Trans. Antennas Propag., vol. 62, no. 9, pp. 4468_4473, Sep. 2014. 